\title{
A new irrationality measure for $\zeta(3)$
}

by

\author{
Masayoshi Hata (Kyoto)
}

1. Introduction and the result. The aim of this paper is to give a new irrationality measure for the number

$$
\zeta(3)=\sum_{n=1}^{\infty} \frac{1}{n^{3}},
$$

as an application of Legendre-type polynomials

$$
L(a, b, c, d ; x)=\frac{x^{a}}{d !}\left(x^{b}(1-x)^{c}\right)^{(d)} \in \mathbb{Z}[x]
$$

of degree $a+b+c-d$, where $a, b, c, d$ are integers satisfying $b, c, d \geq 0$, $b+c \geq d$ and $a \geq \min \{0, d-b\}$.

The irrationality of $\zeta(3)$ was first shown by R. Apéry [1] in 1978. F. Beukers [2] reconstructed Apéry's rational approximation to $\zeta(3)$ by introducing the triple improper integral

$$
\iiint_{B} \frac{L_{n}(x) L_{n}(y)}{1-(1-x y) u} d x d y d u
$$

where $B=(0,1)^{3}$ is the open unit cube and $L_{n}(x)=L(0, n, n, n ; x)$ is the usual Legendre polynomial of degree $n$.

Various proofs of the irrationality of $\zeta(3)$ are known. V. N. Sorokin [9] constructed a number of Hermite-Padé approximations to certain series which lead to the irrationality of $\zeta(3)$. Yu. V. Nesterenko [7], inspired by L. A. Gutnik's work [4], obtained a new continued fraction expansion of $\zeta(3)$ by using the so-called Meyer functions. M. Prévost [8] recovered Apéry's sequences using Padé approximations to the asymptotic expansion of the partial sum of $\zeta(3)$. We note that all the approximations mentioned above give the same irrationality measure

$$
\mu_{0}=1+\frac{4 \log (\sqrt{2}+1)+3}{4 \log (\sqrt{2}+1)-3}=13.4178202 \ldots
$$

1991 Mathematics Subject Classification: Primary 11J82. 
for $\zeta(3)$. Nothing is known about arithmetical properties of the values of Riemann zeta function at odd points greater than 3 .

The above irrationality measure $\mu_{0}$ was improved by R. Dvornicich and C. Viola [3] to $\mu_{1}=12.74359 \ldots$ The author [5] obtained a fairly improved measure $\mu_{2}=8.8302837 \ldots$ by introducing the Legendre-type polynomials

$$
L(0, n-m, n+m, n ; x)=\frac{1}{n !}\left(x^{n-m}(1-x)^{n+m}\right)^{(n)}
$$

of degree $n$, where $m \in[1, n]$ is an integral parameter. This polynomial, regarded as a perturbation of the Legendre polynomial, has the advantage of possessing a large common factor of its coefficients and gives in fact the best known irrationality measure, for example, of $\log 2$.

In this paper, as another perturbation of the Legendre polynomial, we consider the following Legendre-type polynomials:

$$
L_{n, m}(x)=L(0, n+m, n, n ; x)=\frac{1}{n !}\left(x^{n+m}(1-x)^{n}\right)^{(n)}
$$

of degree $n+m$, where $m$ is a positive integral parameter. It then follows from Lemma 2.1 of [5] that $L_{n, m}(x)$ is uniquely determined, up to a multiplicative non-zero constant, by the following three conditions:

(a) $\int_{0}^{1} x^{j} L_{n, m}(x) d x=0$ for $0 \leq j<n$;

(b) $L_{n, m}(x)$ vanishes at the origin with order at least $m$;

(c) $\operatorname{deg} L_{n, m}=n+m$.

Note that the coefficients of $L_{n, m}(x)$ have no common prime factors greater than $\sqrt{2 n+m}$; so it seems to be hopeless to use these polynomials in the study of the irrationality measure for $\log 2$. Nevertheless $L_{n, m}(x)$ is suitable for improving the irrationality measure for $\zeta(3)$. Indeed we have

TheOREM 1. For any $\varepsilon>0$ there exists an effective constant $q_{0}(\varepsilon)$ such that

$$
\left|\zeta(3)-\frac{p}{q}\right| \geq q^{-7.377956 \ldots-\varepsilon}
$$

for any integers $p$ and $q$ satisfying $q \geq q_{0}(\varepsilon)$. The exact value of the constant $\mu=7.377956 \ldots$ is given by

$$
\mu=1+\frac{6 \log c_{0}+d_{0}}{6 \log c_{0}-d_{0}}
$$

where

$$
c_{0}=\frac{352+133 \sqrt{7}}{9} \quad \text { and } \quad d_{0}=26+\pi\left\{\sqrt{3}-\cot \frac{\pi}{9}-\cot \frac{2 \pi}{9}\right\} .
$$

To prove this theorem we need a few lemmas given in the next section. 
2. Preliminaries. The following lemma is due to Beukers [2, Lemma 1].

Lemma 2. For any non-negative integers $r$ and $s$, we have

$$
\begin{aligned}
\iiint_{B} \frac{x^{r} y^{s}}{1-(1-x y) u} d x d y d u-2 \delta_{r, s} \zeta(3) & \text { if } r=s, \\
& = \begin{cases}-\sum_{l=1}^{r} \frac{2}{l^{3}} & \text { if } r \neq s, \\
\sum_{l=\min \{r, s\}+1}^{\max \{r, s\}} \frac{1}{|r-s| l^{2}} & \end{cases}
\end{aligned}
$$

where $\delta_{r, s}$ is Kronecker's delta.

It immediately follows from the above lemma that the integral

$$
I=\iiint_{B} \frac{P(x) Q(y)}{1-(1-x y) u} d x d y d u,
$$

can always be written in the form $\alpha \zeta(3)+\beta$ with $\alpha \in \mathbb{Z}$ and $\beta \in \mathbb{Q}$ for any polynomials $P(x), Q(x) \in \mathbb{Z}[x]$. We call $\beta$ the rational part of the integral $I$, which is uniquely determined by the irrationality of $\zeta(3)$. Thus we have the possibility of improving the irrationality measure for $\zeta(3)$ by choosing suitable polynomials $P(x)$ and $Q(y)$. However this is not an easy task because the corresponding measure depends highly both on the asymptotic decay of the remainder term and on the arithmetical properties of the rational part.

The factor $r-s$ in the denominators on the right-hand side when $r \neq s$ in Lemma 2 is very important. For, if one of the denominators is a multiple of $p^{3}$ for some prime $p$ satisfying $p^{2}>\max \{r, s\}$, then obviously $r \equiv s$ $(\bmod p)$. And this enables us to obtain some arithmetical information on the rational part. The details will be discussed in Section 3 .

Let $D_{n}$ be the least common multiple of $\{1, \ldots, n\}$. Suppose now that $0 \leq \operatorname{deg} P-\operatorname{deg} Q \leq \operatorname{ord}_{0} Q$ where $\operatorname{ord}_{0} Q$ denotes the order of the zero of $Q(x)$ at the origin. Then it follows from Lemma 2 that the rational part $\beta$ of $I$ belongs to $\mathbb{Z} /\left(D_{\operatorname{deg} P}^{2} D_{\operatorname{deg} Q}\right)$, since $|r-s| \leq \max \left\{\operatorname{deg} P-\operatorname{ord}_{0} Q, \operatorname{deg} Q\right\}=$ $\operatorname{deg} Q$. Furthermore this can be sharpened if either $P(x)$ or $Q(y)$ is a specific Legendre-type polynomial, as follows.

Lemma 3. Suppose that $P(x), Q(x) \in \mathbb{Z}[x]$ satisfy $0 \leq \operatorname{deg} P-\operatorname{deg} Q \leq$ $\operatorname{ord}_{0} Q$. Suppose further that either

(1) $P(x)=L(a, b, c, d ; x)$ with $\operatorname{deg} P-\operatorname{deg} Q \leq a \leq d \leq \operatorname{deg} Q$, or

(2) $\quad Q(y)=L\left(a^{\prime}, b^{\prime}, c^{\prime}, d^{\prime} ; y\right)$ with $\operatorname{deg} P-\operatorname{deg} Q \leq \min \left\{a^{\prime}, a^{\prime}+b^{\prime}-d^{\prime}\right\}$ and $a^{\prime} \leq d^{\prime} \leq c^{\prime}$. 
Then the rational part $\beta$ of the integral

$$
I=\iiint_{B} \frac{P(x) Q(y)}{1-(1-x y) u} d x d y d u
$$

belongs to the set

$$
\frac{\Delta}{D_{\operatorname{deg} P}^{2} D_{\operatorname{deg} Q}} \mathbb{Z}
$$

where $\Delta$ is the product of all primes lying in the interval $(\operatorname{deg} Q, \operatorname{deg} P]$.

Proof. Put $P(x)=\sum a_{r} x^{r}$ and $Q(y)=\sum b_{s} y^{s}$. For any prime $p \in$ ( $\operatorname{deg} Q, \operatorname{deg} P]$ one of the denominators on the right-hand side in Lemma 2 is a multiple of $p^{2}$ if and only if $r \geq p$, since $\max \{s,|r-s|\} \leq \operatorname{deg} Q$ for any $r$ and $s$. More precisely, the sum of the coefficients of $p^{-2}$ is equal to

$$
J_{p}=\sum_{r \geq p} \sum_{s} \frac{a_{r} b_{s}}{r-s} .
$$

If (1) holds, then clearly

$$
a_{r}=(-1)^{r-a-b+d}\left(\begin{array}{c}
c \\
r-a-b+d
\end{array}\right)\left(\begin{array}{c}
r-a+d \\
d
\end{array}\right),
$$

which is a multiple of $p$ for any $r \geq p$ since $d \leq \operatorname{deg} Q<p \leq r \leq r-a+d$ and $r-a \leq \operatorname{deg} P-(\operatorname{deg} P-\operatorname{deg} Q)=\operatorname{deg} Q<p$.

On the other hand, if (2) holds, then

$$
\begin{aligned}
\sum_{s} \frac{b_{s}}{r-s} & =\int_{0}^{1} x^{r-1} Q\left(\frac{1}{x}\right) d x=\int_{1}^{\infty} t^{-r-1} Q(t) d t \\
& =\frac{1}{d^{\prime} !} \int_{1}^{\infty} t^{-r+a^{\prime}-1}\left(t^{b^{\prime}}(1-t)^{c^{\prime}}\right)^{\left(d^{\prime}\right)} d t \\
& =\left(\begin{array}{c}
r-a^{\prime}+d^{\prime} \\
d^{\prime}
\end{array}\right) \int_{1}^{\infty} t^{-r+a^{\prime}+b^{\prime}-d^{\prime}-1}(1-t)^{c^{\prime}} d t \\
& =\left(\begin{array}{c}
r-a^{\prime}+d^{\prime} \\
d^{\prime}
\end{array}\right) \int_{0}^{1} x^{r-a^{\prime}-b^{\prime}-c^{\prime}+d^{\prime}-1}(x-1)^{c^{\prime}} d x .
\end{aligned}
$$

Since $r-a^{\prime}-b^{\prime}+d^{\prime} \leq \operatorname{deg} P-a^{\prime}-b^{\prime}+d^{\prime} \leq \operatorname{deg} Q$, the denominator of $J_{p}$ is a divisor of $D_{\operatorname{deg} Q}$. The numerator of $J_{p}$ is a multiple of $p$, since $d^{\prime} \leq$ $a^{\prime}+b^{\prime}-\operatorname{deg} P+\operatorname{deg} Q \leq a^{\prime}+b^{\prime}=\operatorname{deg} Q-c^{\prime}+d^{\prime} \leq \operatorname{deg} Q<p \leq r \leq r-a^{\prime}+d^{\prime}$ and since $r-a^{\prime} \leq \operatorname{deg} P-(\operatorname{deg} P-\operatorname{deg} Q)=\operatorname{deg} Q<p$.

In either case it follows that the integer $D_{\operatorname{deg} Q} J_{p}$ is a multiple of $p$; therefore the integer $D_{\operatorname{deg} P}^{2} D_{\operatorname{deg} Q} \beta$ is a multiple of $\Delta$. This completes the proof. 
3. Construction of the approximations. We consider the integral

$$
I_{n, m}=\iiint_{B} \frac{x^{m} L_{n, m}(x) L_{n, m}(y)}{1-(1-x y) u} d x d y d u,
$$

where $m=[\lambda n]$ and $\lambda \in[0,1)$ is a real parameter. This integral can be written as $\alpha_{n, m} \zeta(3)+\beta_{n, m}$ by Lemma 2 . Putting $P(x)=x^{m} L_{n, m}(x)$ and $Q(y)=L_{n, m}(y)$, we have $\operatorname{deg} P-\operatorname{deg} Q=m=\operatorname{ord}_{0} Q$. Moreover since $P(x)=L(m, n+m, n, n ; x)$ satisfies condition (1) in Lemma 3, the rational part $\beta_{n, m}$ of $I_{n, m}$ belongs to the set

$$
\frac{\Delta_{n}}{D_{n+2 m}^{2} D_{n+m}} \mathbb{Z}
$$

where $\Delta_{n}$ is the product of all primes lying in $(n+m, n+2 m]$.

We put $P(x)=\sum a_{r} x^{r}$ and $Q(y)=\sum b_{s} y^{s}$; that is,

$a_{r}=(-1)^{r}\left(\begin{array}{c}n \\ r-2 m\end{array}\right)\left(\begin{array}{c}n-m+r \\ n\end{array}\right)$ and $b_{s}=(-1)^{s+m}\left(\begin{array}{c}n \\ s-m\end{array}\right)\left(\begin{array}{c}n+s \\ n\end{array}\right)$.

Then we have $\beta_{n, m}=(-1)^{m}\left(-2 \beta+\beta^{\prime}\right)$, where

$$
\beta=\sum_{r=2 m}^{n+m}\left(\begin{array}{c}
n \\
r-2 m
\end{array}\right)\left(\begin{array}{c}
n-m+r \\
n
\end{array}\right)\left(\begin{array}{c}
n \\
r-m
\end{array}\right)\left(\begin{array}{c}
n+r \\
n
\end{array}\right) \sum_{l=1}^{r} \frac{1}{l^{3}}
$$

and

$$
\begin{array}{r}
\beta^{\prime}=\sum_{\substack{r \neq s \\
2 m \leq r \leq n+2 m \\
m \leq s \leq n+m}}(-1)^{r+s}\left(\begin{array}{c}
n \\
r-2 m
\end{array}\right)\left(\begin{array}{c}
n-m+r \\
n
\end{array}\right)\left(\begin{array}{c}
n \\
s-m
\end{array}\right)\left(\begin{array}{c}
n+s \\
n
\end{array}\right) \\
\times \sum_{l=\min \{r, s\}+1}^{\max \{r, s\}} \frac{1}{|r-s| l^{2}} .
\end{array}
$$

Let $\omega=\{n / p\}, \eta=\{m / p\}, \theta_{r}=\{r / p\}$ and $\theta_{s}=\{s / p\}$ for brevity, where $\{x\}$ denotes the fractional part of $x$. Suppose now that one of the denominators on the right-hand side in (4) is a multiple of $p^{3}$ for some prime $p \in[\sqrt{3 n}, n+m]$. Then clearly $r \equiv s(\bmod p)$; hence $\theta_{r}=\theta_{s}$. Put

$$
\begin{aligned}
& S_{1}=\{0 \leq \theta<1: \omega \geq\{\theta-2 \eta\}\}, \\
& S_{2}=\{0 \leq \theta<1: \omega+\{\theta-\eta\}<1\}, \\
& S_{3}=\{0 \leq \theta<1: \omega \geq\{\theta-\eta\}\}, \\
& S_{4}=[0,1-\omega) .
\end{aligned}
$$

For any prime $p>\sqrt{n}$, the exponent of $p$ in the factorization of $\left(\begin{array}{c}n \\ r-2 m\end{array}\right)$ into prime powers is $[n / p]-[(r-2 m) / p]-[(n+2 m-r) / p]=\left\{\theta_{r}-2 \eta\right\}+$ $\left\{\omega+2 \eta-\theta_{r}\right\}-\omega$, and therefore is 0 if $\omega \geq\left\{\theta_{r}-2 \eta\right\}$, and 1 if $\omega<$ 
$\left\{\theta_{r}-2 \eta\right\}$. Thus $p \mid\left(\begin{array}{c}n \\ r-2 m\end{array}\right)$ if and only if $\theta_{r} \notin S_{1}$. The other binomial coefficients $\left(\begin{array}{c}n-m+r \\ n\end{array}\right),\left(\begin{array}{c}n \\ s-m\end{array}\right)$ and $\left(\begin{array}{c}n+s \\ n\end{array}\right)$ satisfy the similar property for $S_{2}, S_{3}$ and $S_{4}$ respectively. Note that $\sum_{j=1}^{4}\left|S_{j}\right|=2$, where $|\cdot|$ denotes the onedimensional Lebesgue measure.

For any subset $S \subset[0,1)$ we define

$$
\sigma_{\eta}(S)=\{0 \leq \theta<1:\{\theta-\eta\} \in S\} ;
$$

that is, $\sigma_{\eta}(S) \equiv \eta+S(\bmod 1)$. Clearly $S_{3}=\sigma_{\eta}([0, \omega])$ and $S_{j}=\sigma_{\eta}\left(S_{j+2}\right)$ for $j=1,2$. We need the following simple lemma.

Lemma 4. For any interval $K \subset[0,1)$ satisfying $|K|<\min \{\eta, 1-\eta\}$, we have

$$
K \cap \sigma_{\eta}(K)=\emptyset .
$$

If , in addition, $K$ is not closed, then the condition $|K|<\min \{\eta, 1-\eta\}$ can be replaced by $|K| \leq \min \{\eta, 1-\eta\}$.

Proof. Suppose that $K \cap \sigma_{\eta}(K) \neq \emptyset$. Then take a point $\xi \in K$ with $\{\xi-\eta\} \in K$. If $\xi \geq \eta$, then $|K| \geq \eta$ since $[\xi-\eta, \xi] \subset K$. Otherwise we have $|K| \geq 1-\eta$ since $[\xi, \xi-\eta+1] \subset K$. Hence $|K| \geq \min \{\eta, 1-\eta\}$. It is clear that $|K|>\min \{\eta, 1-\eta\}$ if $K$ is not closed.

We now distinguish four cases, as follows.

CASE I: $2 \omega+\eta<1$. Obviously $S_{3}=[\eta, \omega+\eta]=S_{3} \cap S_{4}$; hence $\left|S_{3} \cap S_{4}\right|=$ $\omega$. Therefore it follows from Lemma 4 that

$$
\bigcap_{j=1}^{4} S_{j}=\left(S_{3} \cap S_{4}\right) \cap \sigma_{\eta}\left(S_{3} \cap S_{4}\right)=\emptyset
$$

if $\omega<\eta$, since $\omega<1-\eta$.

CASE II: $\omega+\eta<1$ and $2 \omega+\eta \geq 1$. We also have $S_{3}=[\eta, \omega+\eta]$; hence $S_{3} \cap S_{4}=[\eta, 1-\omega)$ and so $\left|S_{3} \cap S_{4}\right|=1-\omega-\eta$. Therefore $\bigcap_{j=1}^{4} S_{j}=\emptyset$ if $\omega+2 \eta \geq 1$, since $1-\omega-\eta \leq 1-\eta$.

CASE III: $\omega+\eta \geq 1$ and $2 \omega+\eta<2$. We then have $S_{3}=[0, \omega+\eta-1] \cup[\eta, 1)$; hence $S_{3} \cap S_{4}=[0, \omega+\eta-1]$ and so $\left|S_{3} \cap S_{4}\right|=\omega+\eta-1$. Thus $\bigcap_{j=1}^{4} S_{j}=\emptyset$ if $\omega+2 \eta<2$, since $\omega+\eta-1<\eta$.

CASE IV: $2 \omega+\eta \geq 2$. We have $S_{3} \cap S_{4}=[0,1-\omega)$; hence $\left|S_{3} \cap S_{4}\right|=1-\omega$. Therefore $\bigcap_{j=1}^{4} S_{j}=\emptyset$ if $\omega \geq \eta$, since $1-\omega \leq \eta$.

We next define

$$
E=\left\{(\eta, \omega) \in[0,1)^{2}: \omega<\min \{\eta, 2-2 \eta\} \text { or } \omega \geq \max \{\eta, 1-2 \eta\}\right\},
$$

which is illustrated in Figure 1. For any $(\eta, \omega) \in E$ we thus have $\bigcap_{j=1}^{4} S_{j}=$ $\emptyset$; hence there exists at least one $j=j(\theta)$ satisfying $\theta \notin S_{j(\theta)}$ for every 


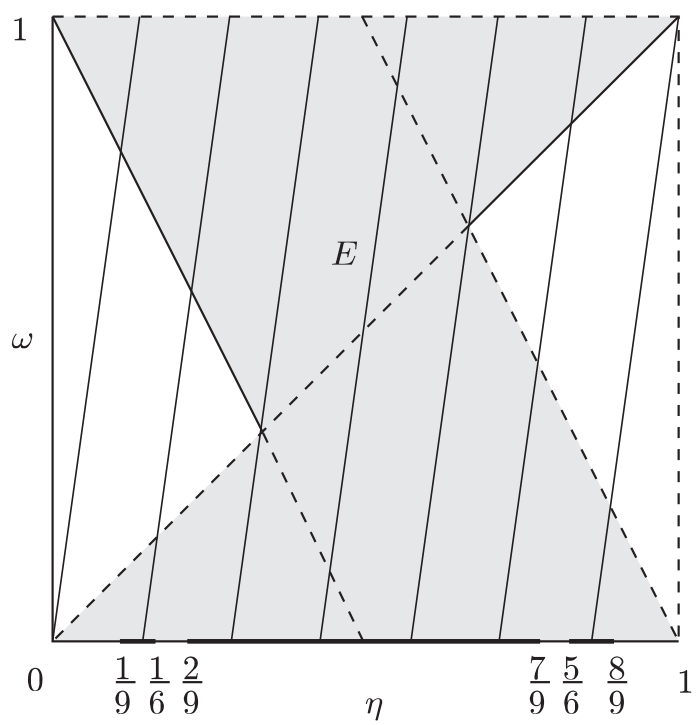

Fig. 1

$\theta \in[0,1)$. This implies that

$$
p \mid\left(\begin{array}{c}
n \\
r-2 m
\end{array}\right)\left(\begin{array}{c}
n-m+r \\
n
\end{array}\right)\left(\begin{array}{c}
n \\
s-m
\end{array}\right)\left(\begin{array}{c}
n+s \\
n
\end{array}\right)
$$

for any $r \neq s$ satisfying $r \equiv s(\bmod p)$; namely, $p \mid D_{n+2 m}^{2} D_{n+m} \beta^{\prime}$. Of course, the same property holds when $r=s$; hence $p \mid D_{n+m}^{3} \beta$ from (3). Therefore $M_{n, m} \beta_{n, m}$ becomes an integer, where

$$
M_{n, m}=\frac{D_{n+2 m}^{2} D_{n+m}}{\Delta_{n} \Delta_{n, m}} \in \mathbb{Z}
$$

and $\Delta_{n, m}$ is the product of all primes $p \in[\sqrt{3 n}, n+m]$ satisfying $(\eta, \omega) \in E$. Thus

$$
M_{n, m} \alpha_{n, m} \zeta(3)+M_{n, m} \beta_{n, m}=M_{n, m} I_{n, m},
$$

our rational approximations to $\zeta(3)$. Note that $\Omega_{n, m} \equiv \Delta_{n} \Delta_{n, m}$ is equal to the product of all primes $p \geq \sqrt{3 n}$ satisfying $(\eta, \omega) \in E$.

4. Proof of Theorem 1. It follows from (5) and Lemma 3.1 of [5] that $\zeta(3)$ has an irrationality measure

$$
\mu \equiv \mu(\lambda)=1+\frac{\sigma(\lambda)+\kappa(\lambda)}{\tau(\lambda)-\kappa(\lambda)}
$$

if $\tau(\lambda)>\kappa(\lambda)$, where

$$
\kappa(\lambda)=\lim _{n \rightarrow \infty} \frac{1}{n} \log M_{n, m},
$$




$$
\sigma(\lambda) \geq \limsup _{n \rightarrow \infty} \frac{1}{n} \log \left|\alpha_{n, m}\right| \quad \text { and } \quad \tau(\lambda)=-\lim _{n \rightarrow \infty} \frac{1}{n} \log \left|I_{n, m}\right| .
$$

We first consider the asymptotic behavior of $M_{n, m}$ as $n \rightarrow \infty$. The prime number theorem implies, analogously to [5], that

$$
\chi(\lambda) \equiv \lim _{n \rightarrow \infty} \frac{1}{n} \log \Omega_{n, m}=\lambda \int_{W} \frac{d t}{t^{2}},
$$

where $W=\{0<t<\infty:(\{t\},\{t / \lambda\}) \in E\}$; hence $\kappa(\lambda)=3+5 \lambda-\chi(\lambda)$. The value of $\chi(\lambda)$ can be easily calculated when $\lambda=1 / k$ for any integer $k \geq 2$. Indeed we have

$$
k \chi\left(\frac{1}{k}\right)=\sum_{l=0}^{\infty} \int_{W_{0}} \frac{d x}{(x+l)^{2}}=\int_{W_{0}} d \psi(x),
$$

where $W_{0}=W \cap(0,1)$ and $\psi(x)=\Gamma^{\prime}(x) / \Gamma(x)$ is the digamma function. Let $H \subset[0,1)^{2}$ be the union of $k$ segments defined by $\omega=\{k \eta\}$. Since the set $\bar{E}$, as well as the set $\bar{H}$, is symmetric with respect to the point $\left(\eta^{*}, \omega^{*}\right)=(1 / 2,1 / 2)$, the set $\pi_{\eta}(\bar{E} \cap \bar{H})$ is also symmetric with respect to $\eta^{*}=1 / 2$ where $\pi_{\eta}(\cdot)$ denotes the orthogonal projection to the $\eta$-axis. This implies that the Stieltjes integral on the right-hand side of (7) can be written as a finite sum

$$
\sum_{j} \varepsilon_{j}\left\{\psi\left(1-\gamma_{j}\right)-\psi\left(\gamma_{j}\right)\right\}
$$

where $\left\{\gamma_{j}\right\}$ are rational numbers which are endpoints lying in $(0,1 / 2)$ of the intervals in $\pi_{\eta}(\bar{E} \cap \bar{H})$ and the sign $\varepsilon_{j}$ is +1 or -1 according as $\gamma_{j}$ is the left or right endpoint. Then using the well-known formula $\psi(1-x)-\psi(x)=$ $\pi \cot (\pi x)$, it follows that

$$
\chi\left(\frac{1}{k}\right)=\frac{\pi}{k} \sum_{j} \varepsilon_{j} \cot \left(\pi \gamma_{j}\right) .
$$

For example, in the case $k=7$, we have $\pi_{\eta}(\bar{E} \cap \bar{H})=[1 / 9,1 / 6] \cup[2 / 9,7 / 9] \cup$ $[5 / 6,8 / 9]$ (see Figure 1); so $\left\{\gamma_{1}, \gamma_{2}, \gamma_{3}\right\}=\{1 / 9,1 / 6,2 / 9\},\left\{\varepsilon_{1}, \varepsilon_{2}, \varepsilon_{3}\right\}=$ $\{+1,-1,+1\}$ and hence

$$
\chi\left(\frac{1}{7}\right)=\frac{\pi}{7}\left\{\cot \frac{\pi}{9}+\cot \frac{2 \pi}{9}-\sqrt{3}\right\} .
$$

Therefore we have $\kappa(1 / 7)=d_{0} / 7$, where $d_{0}$ is the constant defined in Theorem 1.

We next consider the asymptotic behavior of $I_{n, m}$ as $n \rightarrow \infty$. After an $n$-fold partial integration with respect to $y$, we get

$$
I_{n, m}=\iiint_{B} \frac{x^{n+m} L_{n, m}(x) y^{n+m}(1-y)^{n} u^{n}}{(1-(1-x y) u)^{n+1}} d x d y d u .
$$


We now need the following convenient lemma. We call

$$
\tau_{d}(u)=(1-u) /(1-d u)
$$

a nice transformation for any $d<1$; it is a homeomorphism on [0,1] and satisfies $\tau_{d} \equiv \tau_{d}^{-1}$. The nice transformation $\tau_{1-x y}(u)$ was used in [2].

Lemma 5. By substituting $v=\tau_{1-x y}(u)$ we have

$$
\int_{0}^{1} \frac{u^{a}(1-u)^{b}}{(1-(1-x y) u)^{c+1}} d u=(x y)^{b-c} \int_{0}^{1} \frac{v^{b}(1-v)^{a}}{(1-(1-x y) v)^{a+b-c+1}} d v
$$

for any non-negative integers $a, b$ and $c$.

The proof is straightforward. It follows from this lemma that

$$
I_{n, m}=\iiint_{B} \frac{x^{m} L_{n, m}(x) y^{m}(1-y)^{n}(1-v)^{n}}{1-(1-x y) v} d x d y d v .
$$

Then, after an $n$-fold partial integration with respect to $x$,

$$
\begin{aligned}
I_{n, m}= & \frac{(-1)^{n}}{n !} \iiint_{B} x^{n+m}(1-x)^{n} \frac{\partial^{n}}{\partial x^{n}}\left(\frac{x^{m}}{1-(1-x y) v}\right) \\
& \times y^{m}(1-y)^{n}(1-v)^{n} d x d y d v \\
& (-1)^{m} \iiint_{B} \frac{x^{n+m}(1-x)^{n} y^{n}(1-y)^{n} v^{n-m}(1-v)^{n+m}}{(1-(1-x y) v)^{n+1}} d x d y d v ;
\end{aligned}
$$

hence we have

$$
\lim _{n \rightarrow \infty} \frac{1}{n} \log \left|I_{n, m}\right|=\max _{0<x, y, v<1} \log F(x, y, v),
$$

where

$$
F(x, y, v)=\frac{x^{1+\lambda}(1-x) y(1-y) v^{1-\lambda}(1-v)^{1+\lambda}}{1-(1-x y) v} .
$$

The above maximum is actually attained at $\left(x^{*}, y^{*}, v^{*}\right)$ where

$$
x^{*}=\frac{\lambda+\lambda^{\prime}}{\lambda+\lambda^{\prime}+1}, \quad y^{*}=\frac{\lambda^{\prime}}{\lambda^{\prime}+1} \quad \text { and } \quad v^{*}=\lambda^{\prime}-\lambda
$$

with $\lambda^{\prime}=\sqrt{(1+\lambda) / 2}$, which is a unique solution of the equations

$$
\frac{\partial F}{\partial x}(x, y, v)=\frac{\partial F}{\partial y}(x, y, v)=\frac{\partial F}{\partial v}(x, y, v)=0
$$

in $(0,1)^{3}$. For $\lambda=1 / 7$ it can be seen that $F\left(x^{*}, y^{*}, v^{*}\right)=c_{0}^{-6 / 7}$, where the constant $c_{0}$ is defined in Theorem 1 . 
We finally consider the asymptotic behavior of

$$
\begin{aligned}
\alpha_{n, m} & =\frac{2}{2 \pi i} \int_{C_{0}} z^{m-1} L_{n, m}(z) L_{n, m}\left(\frac{1}{z}\right) d z \\
& =\frac{2}{(2 \pi i)^{3}} \int_{C_{0}} \int_{C_{1}} \int_{C_{2}} z^{m-1} \frac{w^{n+m}(1-w)^{n}}{(w-z)^{n+1}} \cdot \frac{\zeta^{n+m}(1-\zeta)^{n}}{(\zeta-1 / z)^{n+1}} d \zeta d w d z
\end{aligned}
$$

as $n \rightarrow \infty$. Taking the contours $C_{0}, C_{1}, C_{2}$ to be the circles centered at $z=0, w=z, \zeta=1 / z$ with radii $r, R, \varrho$ respectively, we get

$$
\limsup _{n \rightarrow \infty} \frac{1}{n} \log \left|\alpha_{n, m}\right| \leq \min _{r, R, \varrho>0} \log G(r, R, \varrho),
$$

where

$$
G(r, R, \varrho)=\frac{(r+R)^{1+\lambda}(1+r+R)(1+r \varrho)^{1+\lambda}(1+r+r \varrho)}{r^{2} R \varrho} .
$$

The above minimum is attained at $\left(r^{*}, R^{*}, \varrho^{*}\right)$ where

$$
r^{*}=\frac{\lambda^{\prime}-\lambda}{\lambda^{\prime}+\lambda}, \quad R^{*}=\frac{2}{\lambda^{\prime}+\lambda}-\frac{1}{\lambda^{\prime}} \quad \text { and } \quad \varrho^{*}=\frac{1}{\lambda^{\prime}-\lambda},
$$

which is a unique solution of the equations

$$
\frac{\partial G}{\partial r}(r, R, \varrho)=\frac{\partial G}{\partial R}(r, R, \varrho)=\frac{\partial G}{\partial \varrho}(r, R, \varrho)=0
$$

in $(0, \infty)^{3}$. Then it can be seen that $F\left(x^{*}, y^{*}, v^{*}\right) G\left(r^{*}, R^{*}, \varrho^{*}\right)=1$; hence we can take $\sigma(\lambda)=\tau(\lambda)$ for any $\lambda$. Therefore $\zeta(3)$ has an irrationality measure

$$
\mu=\mu\left(\frac{1}{7}\right)=1+\frac{6 \log c_{0}+d_{0}}{6 \log c_{0}-d_{0}}
$$

which completes the proof of Theorem 1.

5. Concluding remarks. Theorem 1 is thus proved by taking $\lambda=1 / 7$. However we do not know whether the irrationality measure $\mu(\lambda)$ in $(6)$ attains its minimum at $\lambda=1 / 7$ as the real parameter $\lambda \in(0,1)$ varies, although numerical calculations seem to support this.

We also note that the integral

$$
\iiint_{B} \frac{x^{m} L_{n}(x) L_{n+m}(y)}{1-(1-x y) u} d x d y d u
$$

gives almost the same irrationality measure $\mu$ as in Theorem 1 numerically. In this case we need to calculate the corresponding Stieltjes integral with respect to the digamma function $\psi(x)$ over twelve intervals, which is more complicated than $W_{0}$. Moreover the diffeomorphism

$$
T(x, y, u)=\left(1-(1-x y) u, \frac{x y}{1-(1-x y) u}, \frac{1-x}{1-x y}\right)
$$


acting on the unit cube $B$ and satisfying $T \equiv T^{-1}$, converts the integral $I_{n, m}$ constructed in Section 3 into

$$
\iiint_{B} \frac{x^{m} L_{n+m}(x) L_{n-m, 2 m}(y)}{1-(1-x y) u} d x d y d u
$$

although this integral representation does not seem to give any further arithmetical information on the rational part $\beta_{n, m}$.

Acknowledgements. The author would like to thank the referee for suggesting many improvements and correcting some minor errors.

\section{References}

[1] R. Apéry, Irrationalité de $\zeta(2)$ et $\zeta(3)$, Astérisque 61 (1979), 11-13.

[2] F. Beukers, A note on the irrationality of $\zeta(2)$ and $\zeta(3)$, Bull. London Math. Soc. 11 (1979), 268-272.

[3] R. Dvornicich and C. Viola, Some remarks on Beukers' integrals, in: Number Theory, Colloq. Math. Soc. János Bolyai 51, North-Holland, 1987, 637-657.

[4] L. A. Gutnik, On the irrationality of some quantities containing $\zeta(3)$, Acta Arith. 42 (1983), 255-264 (in Russian); English transl.: Amer. Math. Soc. Transl. 140 (1988), $45-55$.

[5] M. Hata, Legendre type polynomials and irrationality measures, J. Reine Angew. Math. 407 (1990), 99-125.

[6] —, A note on Beukers' integral, J. Austral. Math. Soc. Ser. A 58 (1995), 143-153.

[7] Yu. V. Nesterenko, A few remarks on $\zeta(3)$, Mat. Zametki 59 (1996), 865-880 (in Russian); English transl.: Math. Notes 59 (1996), 625-636.

[8] M. Prévost, A new proof of the irrationality of $\zeta(2)$ and $\zeta(3)$ using Padé approximants, J. Comput. Appl. Math. 67 (1996), 219-235.

[9] V. N. Sorokin, Hermite-Padé approximations for Nikishin systems and the irrationality of $\zeta(3)$, Uspekhi Mat. Nauk 49 (1994), no. 2, 167-168 (in Russian); English transl.: Russian Math. Surveys 49 (1994), no. 2, 176-177.

Division of Mathematics

Faculty of Integrated Human Studies

Kyoto University

Kyoto 606-8501, Japan

E-mail: hata@i.h.kyoto-u.ac.jp 